\title{
A novel method to cease traumatic urethral bleeding
}

elSSN: 2383-4625

\author{
Hamed Akhavizadegan \\ Department of Urology, Baharloo Hospital, Tehran University of Medical Sciences, Tehran, Iran
}

Urethral bleeding due to trauma is a well-studied and often self-limiting condition. However, existing evidence regarding effective management of massive hemorrhage is limited. Intermittent penile compression and continuous perineal pressure are methods commonly used to control urethral bleeding. Nevertheless, these methods are not mentioned in the literature as specific treatment for this condition, and are rather learned by physicians via word of mouth. Sometimes these methods are ineffective and difficult for the patient to tolerate. This paper explains a new method implemented on a young man with massive urethral bleeding due to iatrogenic trauma, which was unresponsive to standard management. For this patient, the bleeding was controlled by gradually inflating the balloon of the catheter up to $4 \mathrm{~mL}$. Given its safety and efficiency in controlling bleeding in this patient, it seems this new technique should be considered in cases of massive hemorrhage unresponsive to routine treatment.

Keywords Urethral stricture; Injuries; Urinary catheters; Hemorrhage

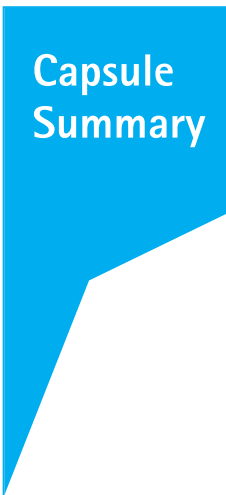

What is already known

Urethral bleeding due to trauma often is a self-limiting complication. However, in massive cases, much is not found in the literature regarding management.

What is new in the current study

Intermittent penile and continuous perineal compression are methods that are taught but not mentioned in the literature directly. Sometimes these methods are ineffective and difficult to tolerate. In a young man with massive urethral bleeding due to iatrogenic trauma which didn't respond to these methods, the bleeding stopped by gradually inflating the balloon of the catheter up to $4 \mathrm{~mL}$.
Received: 3 October 2017

Revised: 1 December 2017

Accepted: 21 January 2018

Correspondence to: Hamed

Akhavizadegan

Department of Urology, Baharloo

Hospital, Behdari Street, RahAhan

Square, Tehran 12299, Iran

E-mail:h-akhavizadegan@tums.ac.ir

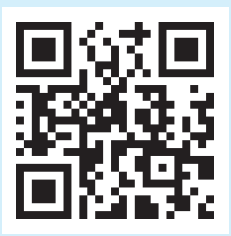

How to cite this article:

Akhavizadegan H. A novel method to cease traumatic urethral bleeding. Clin Exp Emerg Med 2018;5(1):66-68.

This is an Open Access article distributed under the terms of the Creative Commons Attribution Non-Commercial License (http:// creativecommons.org/licenses/by-nc/4.0/). 


\section{INTRODUCTION}

Compression of the hemorrhaging site is known as the traditional and commonly used method to control bleeding. Although easy and effective in superficial bleeding, it may not be helpful in controlling internal bleeding. The bulbar urethra can be considered superficial; however, its location in the perineum makes it difficult to maintain pressure on the bulbar urethra. Thus, the main way to control bulbar urethral bleeding is to insert a urethral indwelling catheter or in case of incapability of passing a catheter, applying pressure on the perineum and intermittent penile urethral compression are recommended. ${ }^{1,2}$ These methods have their own drawbacks. Herein, a novel method to control traumatic urethral bleeding is introduced.

\section{CASE REPORT}

A 24-year-old male patient with a one-centimeter bulbar stricture due to pelvic fracture was a candidate for internal urethrotomy in Baharloo Hospital, affiliated with Tehran University of Medical Sciences. Because of acceptable lumen and short length of stricture, general anesthesia with mask was selected to ensure a minimum operating time. Upon reaching into the stricture site, the patient moved inadvertently due to inadequate anesthesia. The urethrotomy blade cut the urethra and caused severe bleeding into the urethra which obscured the vision. The procedure was terminated and suprapubic percutaneous cystostomy was performed. The urethral bleeding was controlled by perineal pressure. Two hours later, bleeding restarted and the patient did not respond to newly applied pressure. Intermittent compression of penile urethra for 20 minutes followed by 5 minutes relief was performed; however, the bleeding continued. A 12-Fr indwelling catheter was inserted gently in to the urethra. When it reached the stricture and resistance was sensed, advancement was stopped. The balloon of urethral catheter was filled with water in 1-mL aliquots: after injecting each milliliter of distilled water to the balloon, we waited for 5 minutes, giving time to control bleeding. After injection of 4-mL water in to the balloon, bleeding ceased. The external end of the urethral catheter was ligated, in order to stop continuous bleeding through the catheter. The patient was more comfortable in comparison to perineal pressure according to his self-report.

The glans of the penis was examined every 15 minutes for the first hour and then every hour for any discoloration. After 6 hours, the catheter was removed and no bleeding was observed. Before attempting to perform internal urethrotomy three months later, we performed a new retrograde urethrography, for which results were exactly as the previous one without any increase in severity or length of stricture. The internal urethrotomy was performed uneventfully.

\section{DISCUSSION}

Urethral trauma can occur due to pelvic and perineal injuries or iatrogenic trauma to the urethra. ${ }^{3}$ Urethral bleeding as one of the complications of urethral trauma is not usually life-threatening, nevertheless it can be very embarrassing. ${ }^{3,4}$ When the urethral catheter can be inserted, bleeding will be controlled gradually in all cases and nothing more is necessary. ${ }^{5}$ The problem is for cases where insertion of a urinary catheter is impossible. Traditionally, direct pressure on the bleeding site is the standard way to control hemorrhage. Putting pressure on the perineum is the only way we can reach this goal. In the perineum, applying a pressure dressing over a solid object which is fixed with adhesive bands to the lower abdomen is the conventional method. ${ }^{1,2}$

In applying this method, providing direct pressure on the distal end of urethra and also corpus spongiosum and urethral arteries ceases the bleeding. ${ }^{2}$ However, the patient is not comfortable as he should not move. Moreover, the adhesive bands lose their tension over time and should be fixed intermittently, which is time consuming. Additionally, sometimes this approach is not effective.

The second method to control traumatic or non-traumatic urethral bleeding is to apply intermittent penile ligation. This intervention does not permit bleeding through the urethra, increases pressure at the bleeding site and helps homeostasis. ${ }^{2}$ In this patient for whom manual pressure on the perineum and intermittent penile compression were not effective in controlling bleed-

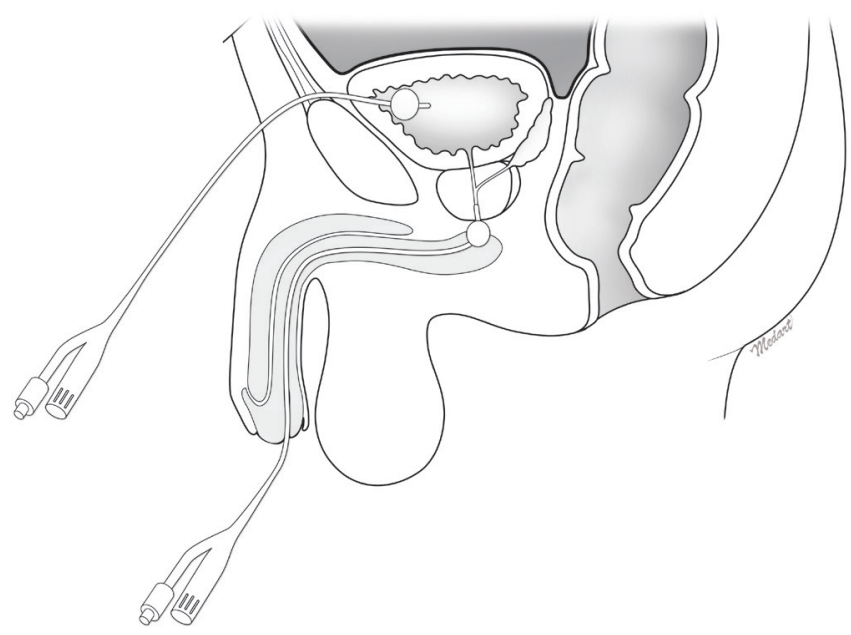

Fig. 1. Schematic diagram of gradual inflation of balloon of urethral catheter in the urethra in order to control bleeding. 


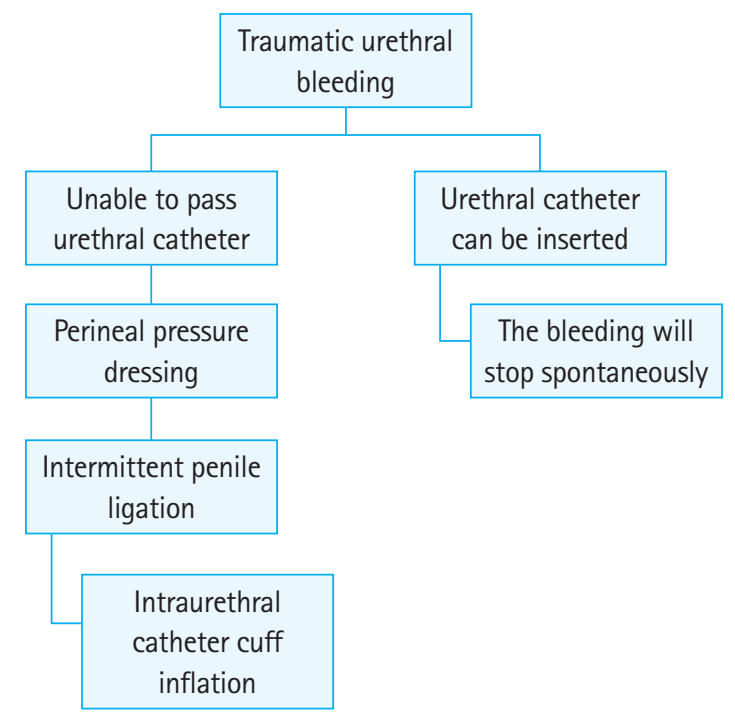

Fig. 2. Algorithmic approach to control of urethral bleeding.

ing, pressure was used by inflating the urinary catheter balloon. Gradually increasing the balloon size prevented further urethral injury. Inflating the balloon just proximal to the bleeding site occluded the urethra and worked as penile ligation without causing penile ischemia (Fig. 1). Moreover, the balloon applied direct pressure on the bleeding site, similar to a perineal pressure dressing.

In our case, bleeding stopped after injection of 4-mL water in $1-\mathrm{mL}$ aliquots. We injected this volume of water in to a 12-Fr indwelling catheter and the diameter of the balloon was $17 \mathrm{~mm}$ (near $50 \mathrm{Fr}$ ). According to the literature, urethral dilatation with a catheter up to $26 \mathrm{Fr}$ is the classic method while our dilatation was about $9 \mathrm{~mm}$ more than this size, in terms of the diameter. ${ }^{6}$

Regarding the potential of causing stricture by the inflated balloon, we compared the retrograde urethrography before and after the iatrogenic injury and there were no additional strictures in the distal part of urethra (place of inflating the balloon), although this possibility cannot be ignored in future cases.

In case of uncontrollable urethral bleeding and inability to cath- eterize, balloon inflation if performed gradually $(1 \mathrm{~mL}$ every $5 \mathrm{~min}$ utes), until bleeding ceases can be an option. Due to the possibility of further urethral injury, it is recommended to use conventional methods first, and to reserve this technique for intractable cases (Fig. 2).

\section{CONFLICT OF INTEREST}

No potential conflict of interest relevant to this article was reported.

\section{ACKNOWLEDGMENTS}

The author is grateful to Mr. Hasan Mirzaie Motlagh, operating room technician, for production of Fig. 1.

\section{REFERENCES}

1. McWilliams WA, Javadpour N, Young JD. Simple method to control bleeding and for urethral continuity in posterior urethral injuries. Urology 1985;26:405-6.

2. Fishman IJ, Perez E. Simple technique for acute management of urethral hemorrhage. Urology 1992;39:294.

3. Mundy AR, Andrich DE. Urethral trauma. Part I: introduction, history, anatomy, pathology, assessment and emergency management. BJU Int 2011;108:310-27.

4. Huang TY, Huang CN, Lee YC. Life-threatening urethral bleeding induced by a pseudoaneurysm of the obturator artery. Urology 2013;82:e43-4.

5. Martinez-Pineiro L, Djakovic N, Plas E, et al. EAU guidelines on urethral trauma. Eur Urol 2010;57:791-803.

6. Akkoc A, Aydin C, Kartalmıs M, Topaktas R, Altin S, Yilmaz Y. Use and outcomes of amplatz renal dilator for treatment of urethral strictures. Int Braz J Urol 2016;42:356-64. 